\title{
CCGA-BN Constructor: A Bayesian Network Learning Approach
}

\author{
Maryam Feroze \\ Department of Computer \\ Science/UBIT, University of \\ Karachi, Pakistan
}

\author{
Muhammad Saeed \\ Department of Computer \\ Science/UBIT, University of \\ Karachi, Pakistan
}

\author{
Nasir Touheed, Ph.D. \\ Faculty of Computer Science, \\ Institute of Business Administration, \\ Karachi, Pakistan
}

\begin{abstract}
This paper presents a tool CCGA-BN Constructor for learning Bayesian network that uses cooperative coevolutionary genetic algorithm to learn Bayesian network structure from data. The problem has been broken down into two sub-problems: (a) to find the optimal nodes'ordering and (b) to find the optimal adjacency matrix of the graph. Both the sub-problems' solutions are then combined to produce the optimal structure. CCGA-BN constructor used Bayesian score for networks having nodes with more than two states and BIC for network having bistate nodes. The findings of this paper are compared against the original structures and the results show a lot of promise.
\end{abstract}

\section{Keywords}

Bayesian network, cooperative co-evolutionary genetic algorithm, structure learning, Bayesian score, BIC.

\section{INTRODUCTION}

Bayesian Network(BN) is a model, which represents and reasons under uncertainty, represented as Directed Acyclic Graph (DAG) whose vertices signifies the domain variables and the edges, if present, establish the conditional dependence between variables.

If defineformallya Bayesian Network $\boldsymbol{N}$ is consists ofa DAG $\mathrm{G}=(\mathrm{V}, \mathrm{E})$ with verticesVand edgesE, a set of discrete random variables, $X=\left\{X_{1}, \ldots, X_{n}\right\}$, represented by the vertices of $G$ and $a$ set of conditional probability distributions(CPDs), $\mathrm{P}$, containing a distribution, $\boldsymbol{P}\left(\boldsymbol{X}_{i} \mid \boldsymbol{p a}\left(\boldsymbol{X}_{i}\right)\right)$, for each $\mathrm{X}_{\mathrm{i}} \in \mathrm{X}$. A Bayesian network decomposes the joint probability distribution $\boldsymbol{p}(\boldsymbol{X})=\boldsymbol{p}$ $\left(\boldsymbol{X}_{1}, \ldots \boldsymbol{X}_{n}\right)$ into a product of conditional probability distributions over each variable given its parents:

$$
p(X)=\prod_{i=1}^{n} p\left(X_{i} \mid p a\left(X_{i}\right)\right)
$$

wherepa $\left(\mathrm{X}_{\mathrm{i}}\right)$ be the set of parents of $\mathrm{Xi}$ in $\mathrm{G}$.

A Bayesian network can be constructed manually with the help of services, skills and knowledge of the domain expert or can be learned from the observed data.The DAG of a BN was constructed by the collaboration of domain experts until the early 1990s and then the conditional probabilities of the DAG were either calculated from the available data or by the wisdom and knowledge of experts were estimated, also the combination of both approaches was practiced. Construction of a BN manually by experts can be a laborious and can take long time especially in the case of large networks. Thus, the researchers proposed methods that enable to learn $\mathrm{BN}$ from the empirical data set. Learning $\mathrm{BN}$ from data is composed of two tasks (i) Learning structure of $\mathrm{BN}$ that is DAG (ii) Learning $\mathrm{BN}$ parameters that is CPDs for each variable. In this paper a method for learning structure of the $\mathrm{BN}$ from the data is used and parameters are then learned in conventional way.

The task of learning DAG of a BN from a data set can be formulated as an optimization problem[1][2] that has been proven to be NP-Hard[3] as learning the DAG from data by exhaustively considering all possibilities is not feasible, regardless of the size of the data, since the possible DAGs that can be constructed grows super-exponentially in the number of vertices.Hence, this either requires search algorithms that uses sub-optimal heuristics or algorithms that produces optimal solution under certain assumptions.

Coevolution is said to be a mutual evolutionary variation between species that interact with each other. Coevolution, based on the method of calculation of the fitness of individuals, can be based either on competition or on cooperation. When the coevolution is competitive, the fitness is computed by direct competition of different species whereas in the cooperation, the fitness of an individual species depends on the collaboration with other species. Cooperative Coevolutionary Genetic Algorithm (CCGA) can be applied to the problems that can be decomposed into number of independent parameters/species that coordinates with each other in order to compute the fitness of the complete individual. Fitness to an individual of a particular species can be assigned by merging it with the selected individuals of the other species to form one complete individual whose fitness can be computed in the normal fashion, which is then used to assign fitness to the individual component. The task of structure learning can be divided into two optimal sub-problems (a) to find an ordering of the nodes that is optimal and (b) to find an optimal connectivity matrix. Thus, the problem can be modeled and solved using CCGA which uses two species one for each subproblem.

Previously, in this field of researchdifferent evolutionary algorithms such as Genetic Algorithms (GA) and its variants have been used [4][5][6][7], all these approaches address this problem as one complete problem of optimal DAG generation from data. Cooperative Coevolutionary Genetic Algorithm is also used in [8] for learning the structure of BN from datasets. In this paper, the problem is representedin the similar way as in [8] but algorithm is a variation of conventional CCGA.

Besides this section, this paper is organized as follows. In the following section, an introduction to Bayesian networks learning, structure learning problem and scoring methods is discussed. In Section 3,we present overview CCGA-BN andin section 4the results obtained for some networks and the comparison of obtained structures with the original ones are discussed. We show that the approach of BN-CCGA performs satisfactory. Finally, we conclude in last section . 


\section{BAYESIAN NETWORK LEARNING 2.1 Parameter Learning}

BN parameters are estimated form the data set in the way that satisfies the following theorem:

Theorem: Suppose we specify a Bayesian network for parameter learning in the case of binomial variables $X_{i}$ and assign for all iand $j$

$$
a_{i j}=b_{i j}=N / 2 q_{i}
$$

whereN is a positive integer and $q_{i}$ is the number of instantiations of the parents of the ithvariable. $a_{i j}$ and $b_{i j}$ specifies the two states of the variable $X_{i}$ and for jth state of the $p a\left(X_{i}\right) . \quad$ Then the resultant Bayesian network has equivalent sample size $N$, and the joint probability distribution in the Bayesian network is uniform.

The prior probability of the variables is calculated as:

$$
\begin{aligned}
& P\left(X_{i}=a_{i j}\right)=a_{i j} /\left(a_{i j}+b_{i j}\right) \\
& P\left(X_{i}=b_{i j}\right)=b_{i j} /\left(a i j+b_{i j}\right)
\end{aligned}
$$

The updated probability calculated from the data set of $M$ elements is:

$$
\begin{aligned}
& P\left(X_{i}=a_{i j}\right)=\left(a_{i j}+s_{i j}\right) /(N+M) \\
& P\left(X_{i}=b_{i j}\right)=\left(b_{i j}+t_{i j}\right) /(N+M)
\end{aligned}
$$

Pair $\left(s_{i j}, t_{i j}\right)$ is used to represent the counts for the ith variable's instances in the data when the variable's parents have their $j$ th value.

For Multinomial Variables $\mathrm{X}_{\mathrm{i}}$, having $\boldsymbol{s}$ states, for its $\boldsymbol{r t h}$ state $a_{r}$ we will have:

$$
a_{r i j}=N / s q_{i}
$$

The prior probability of $X_{i}$ is:

$$
P\left(X_{i}=a_{r i j}\right)=a_{r i j} / \sum_{r=1}^{s} a_{r i j}
$$

\subsection{Structure Learning}

The problem of structure learning is to learn a DAG from data that fulfills the Markov condition with the probability distribution $\mathrm{P}$ that is generating the data, where $\mathrm{P}$ is not known. Thispracticeof learning such a DAG is called model selection.

Bayesian Network $N$ with DAG $G$ and Probability distribution $P$ satisfies the Markov condition if and only if $P$ is equal to the product of its conditional distributions of all nodes given their parents in $G$, whenever these conditional distributions exist.

Approaches to learning the structure of a Bayesian network have been classified into three classes [9]. The constrainedbased approach derives set of (in)dependencies from the data that are used in learning structure as constraints [10]. The score-based approach calculates a scoring function for the structures that are learned from the data, the scoring function is the measure of the fitness of structure on the observed data.Lastly, the Bayesian model averaging is a collaborative approach that generates structure from the integration of different structures and inference is performed by averaging the results provided from different models learned. In this paper, the focus is on the approach of score-based learning.

There are two common approaches in score-based approach of structure learning, one is the Bayesian scoring and the other one is Bayesian Information Criterion Score. In this paper both of the scoring approaches are used, for network with only bi-state variables BIC is used to learn the best DAG G, and for data having nodes with more than two states Bayesian Scoring is used.

\subsection{Score based Learning}

In score-based structure learning, scoring function $\Phi$ is considered to quantify how well the DAG fits the data. Our problem can be defined as "Given a data $D=\left\{y_{1}, \ldots, y_{N}\right\}$ and a scoring function $\Phi$, we need to find a $D A G G$ that maximizes the value $\Phi(G, D)$ ".

A Bayesian score function $\Phi$, isvalued by evaluation of the posterior probability of a graph $\mathrm{G}$ given the data $D$ :

$$
\begin{aligned}
& P(G \mid D)=\frac{P(D \mid G) P(G)}{P(D)} \\
& P(G \mid D) \propto P(D \mid G) P(G)
\end{aligned}
$$

where the equality is given by using Bayes' theorem. The denominator in above equation doesn't help in distinguishing between the different structures and can be disregarded as it is simply a normalizing factor. Similarly $P(G)$ of each possible $G$ for the data is same, thus $P(D \mid G)$ is only what we need to compute for scoring.

For variables $X_{i}[2]$ proposed the use of a Dirichlet parameter prior for all parameters in the network, then $P(D \mid \mathrm{G})$ can be obtained as:

$$
P(D \mid G)=\prod_{i=1}^{n} \prod_{j=1}^{q_{i}} \frac{\Gamma\left(N_{i j}^{\prime}\right)}{\Gamma\left(N^{\prime}{ }_{i j}+N_{i j}\right)} \prod_{k=1}^{r_{i}} \frac{\Gamma\left(N_{i j k}^{\prime}+N_{i j k}\right)}{\Gamma\left(N_{i j k}^{\prime}\right)}
$$

where $n$ represents the count of variables in the network, $r_{i}$ is the count of all possible values for the variable $X_{i}, q_{i}$ is the total number of possible joint assignment of values to the parents of $X_{i}, N_{i j k}$ is the number of occurrences of configurations of variables and their parents, $N^{\prime}{ }_{i j k}$ are the prior counts of occurrences of variables and their parents calculated as $N_{i j}=\sum_{k=1}^{r_{i}} N_{i j k}$ and $N_{i j}^{\prime}=\sum_{k=1}^{r_{i}} N_{i j k}^{\prime}$, and $\Gamma$ is the Gamma function, which satisfies $\Gamma(m)=(m-1)$ !. The logarithm of equation is more practical to use since it is more manageable to compute numerically.

The total number of possible structures $r(n)$ for a network with $n$ nodes, can be found by the recursive formula[11]:

$$
r(n)=\sum_{k=1}^{n}(-1)^{k+1}\left(\begin{array}{l}
n \\
k
\end{array}\right) 2^{k(n-k)} r(n-k)
$$

i.e., $r(n)$, which is the function based on the number of nodes, is super-exponential in $n$. When $n=6, r(n)=3,781,503$, i.e.this many possible DAGs. This result reaches approximately $4.2 \times 1018$ when $n=10$.

\subsection{Bayesian Information Criterion}

Another common scoring criterion for BN structure learning is Bayesian information criterion (BIC) scores, which is as follows:

$$
B I C(G: D)=\ln (P(D \mid \widehat{P}, G))-\frac{d}{2} \ln m
$$

where $\mathrm{m}$ is the number of data items, $\mathrm{d}$ is the number of parametersof the DAG model, and $\hat{P}$ is the set of maximum likelihood values of the parameters. 


\section{BN-CCGA CONSTRUCTOR}

The proposed solution to the Bayesian network structure learning problem from a fully observable data set is established on the cooperative coevolutionary genetic algorithm (CCGA) proposed by Potter and De Jong [12]. In this solution onecomplete problem is divided into two dependent subproblems(a) to find an optimal ordering of the nodes and (b) to find an optimal connectivity matrix.

Therefore, two independent species,each representing a different but related sub-problem, are required that can collaborate and cooperate with each other to form a complete solution, one species to represent each subproblem. The algorithm, of CCGA-BN is given in figure

1. Initialize $\mathrm{n}$ individuals of Species 1

2. Initialize $\mathrm{n}$ individuals of Species 2

3. For $m$ generations performs the following steps:

3.1. For both species perform following steps to produce $\mathrm{n}$ individuals

3.1.1. Select two individuals/parents $\mathrm{P} 1, \mathrm{P} 2$

3.1.2. $\mathrm{P} 1$ and $\mathrm{P} 2$ undergoes Crossover to produce $\mathrm{C} 1$ and $\mathrm{C} 2$

3.1.3. Mutate $\mathrm{C} 1$ and $\mathrm{C} 2$

3.1.4. $\mathrm{C} 1$ from both species are united to produce one complete solution individual $\mathrm{I} 1$ and $\mathrm{C} 2$ from both species are combined to produce complete solution individual I2

3.1.5. Evaluate I1 and I2

3.2. New generation of both species are built by replacing the individuals of previous generation with the newly created fittest individuals.

4. Best Individual of the last generation gives the solution

Each individual in species 1 , which can also be named as permutation population, is represented by ordering of the random variables $X_{1}, X_{2}, \ldots, X_{n}$, where parent nodes of the node present at position $i$ come at any position between 1 and i-1. A fully connected Bayesian network with $n$ nodes has $(n-1) n / 2$ edges. An individual from the binary subpopulation is represented string $b_{1,2}, b_{1,3}, \ldots, b_{1, n}, b_{2,3}, \ldots, b_{2, w} \ldots$, $\boldsymbol{b}_{\boldsymbol{n}-\mathbf{1}, \boldsymbol{n}}$; where $b_{i, j}$ is 1 if the node at position $i$ is a parent of the node at position $\mathrm{j}$, and is 0 otherwise. The length of the string is $(n-1) n / 2$. Also it can be visualized as for $n=4$ in the table1 below.

Table 1: Species 2 Individual Representation

\begin{tabular}{|l|l|l|l|l|}
\hline & 1 & 2 & 3 & 4 \\
\hline 1 & 0 & $b_{1,2}$ & $b_{1,3}$ & $b_{1,4}$ \\
\hline
\end{tabular}

\begin{tabular}{|l|l|l|l|l|}
\hline 2 & 0 & 0 & $\mathrm{~b}_{2,3}$ & $\mathrm{~b}_{2,4}$ \\
\hline 3 & 0 & 0 & 0 & $\mathrm{~b}_{3,4}$ \\
\hline 4 & 0 & 0 & 0 & 0 \\
\hline
\end{tabular}

One complete solution i.e. structure of $\mathrm{BN}$ is formed by integrating one individual from both the populations in such a way that for each element at position $i$ in the permutation population individualrepresents a node that is parent of the node appearing at position $i+x(\mathrm{x}<=$ number of node) if there is $c_{i, i+x}=1$ in binary population individual. For example, if permutation population individual is $\mathrm{D}, \mathrm{B}, \mathrm{A}, \mathrm{C}$ and binary population individual is $1,0,1,1,0,1$ then the solution is the DAG with an edge from $D$ to $B$ and $C$, from $B$ to $A$ and from A to $\mathrm{C}$.

The solution obtained will always be legal and will never have cycles because node ordering is implicitly specified and only the strictly upper triangular connectivity matrix is used to represent connectivity. Also, the solution is complete because every possible structure of Bayesian network can be represented.

Remaining details of the algorithm are summarizedin table 2given below.

Table 2: CCGA Details

\begin{tabular}{|c|c|}
\hline Fitness Function & $\begin{array}{l}\text { Фfor muti-valued variables } \\
\text { BIC for bi-valued variables }\end{array}$ \\
\hline \multicolumn{2}{|c|}{ SPECIES 1 -Nodes Ordering } \\
\hline Representation & $\begin{array}{l}\text { Permutation Population representing } \\
\text { ordering of the nodes }\end{array}$ \\
\hline Initialization & Random initialization \\
\hline Selection & Tournament Selection \\
\hline Crossover & Cyclic Crossover with probability $\mathrm{p}_{\mathrm{c}}$ \\
\hline Mutation & Swap mutation with probability $\mathrm{p}_{\mathrm{mp}}$ \\
\hline Replacement & Elicit replacement \\
\hline \multicolumn{2}{|c|}{ SPECIES 2 - Connectivity Matrix } \\
\hline Representation & $\begin{array}{l}\text { Binary Population representing } \\
\text { connectivity matrix }\end{array}$ \\
\hline Initialization & Semi-random initialization \\
\hline Selection & Tournament Selection \\
\hline Crossover & $\begin{array}{l}\text { Two-point crossover with probability } \\
\mathrm{p}_{\mathrm{c}}\end{array}$ \\
\hline Mutation & Bit-flip with probability $\mathrm{p}_{\mathrm{mb}}$ \\
\hline Replacement & Elicit replacement \\
\hline
\end{tabular}


By using the above mentioned approach CCGA-BN Constructor is built with the parameters of algorithms discussed in the later section. CCGA-BN Constructor is implemented on language C\#. Also, Smile API is used to produce output network in any of the following formats: xdsl, dsl (genie files), dne (Netica File), net (Hugin File), erg (Ergo File), dsc (Interchange). CCGA BNC reads data file from either the text document or from any of the two formats of excel files and then uses this dataset to perform the task of $\mathrm{BN}$ learning by using Bayesian score for multi-state variable network and uses BIC for networks with variables having only two states.

\section{EXPERIMENTS}

The experiment is performed on multiple networks and here analysis is presented on two of those networks. One of the two networks is composed of 9 nodes and the other one is a well-known Alarm Network.

Parameters of used in CCGA-BN Constructor are in table3.

Table 3: Parameters of CCGA

\begin{tabular}{|l|l|}
\hline \multicolumn{1}{|c|}{ Parameter } & \multicolumn{1}{c|}{ Value } \\
\hline Total generations & 100 \\
\hline Population size & 100 \\
\hline $\mathrm{P}_{\mathrm{mb}}$ & $1 /[\mathrm{n}(\mathrm{n}-1) / 2]$ \\
\hline $\mathrm{P}_{\mathrm{mp}}$ & 0.5 \\
\hline $\mathrm{P}_{\mathrm{c}}$ & 0.6 \\
\hline
\end{tabular}

Where $n$ is the total number of nodes.

For both Bayesian networks, first the data sets containing 1000 instances are generated and then CCGA-BN Constructor for learning structure is executed. Network 1 for which CCGA-BN Constructor was executed is given below:

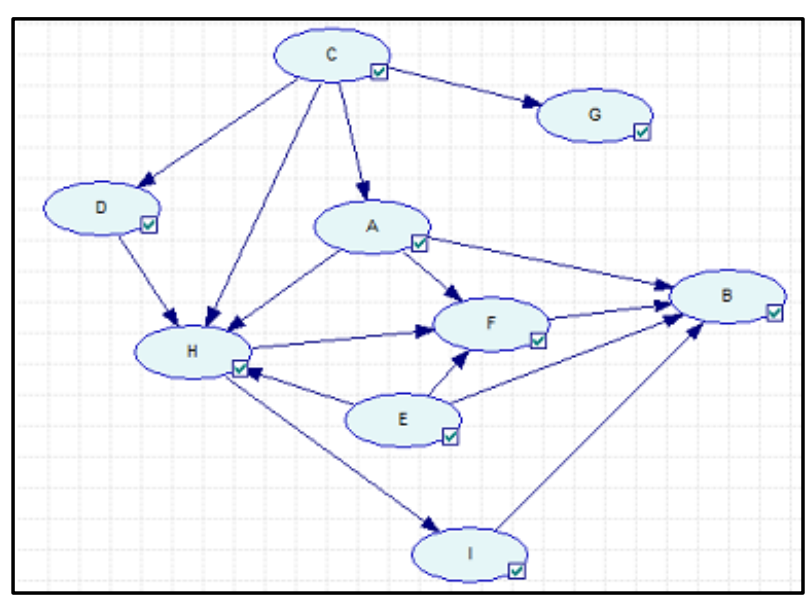

Figure 1: Network 1

The structure learned from CCGA for Network 1is shown in Figure 3.

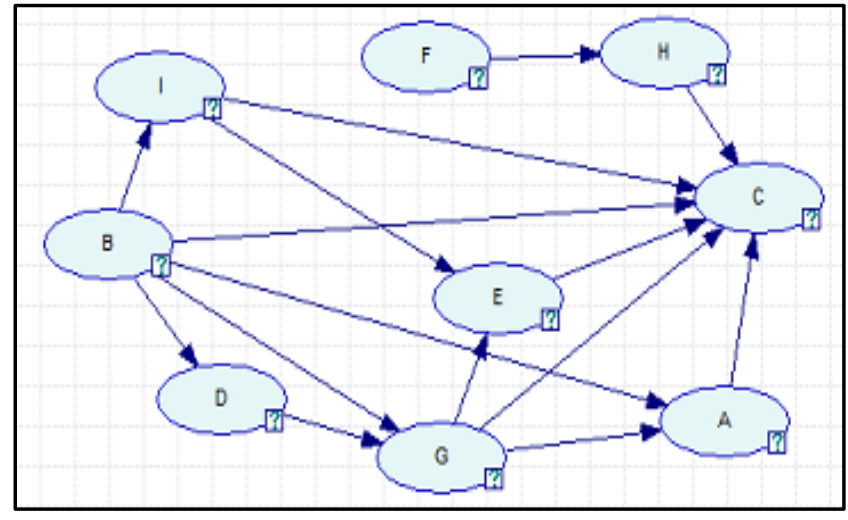

Figure 2: Resultant Network

For this network, the resultant network and the original one don't have any common edge, no converging relationship is maintained i.e. the resultant network doesn't seems to be a good output if sustaining of connections and relationships is to be considered. But if the two networks are compared on the basis of their marginal probabilities and on the inferences when 1, 2 and 3 evidences are set on some random nodes, the result is quite satisfactory. That is, the differences in the marginal probabilities are minor for most of the nodes. The comparison of these probabilities of network 1 is given in the following tables.

Table 4: Comparison of the network 1 with the resultant learned network from the CCGA-BN Constructor on the basis of Marginal Probabilities

\begin{tabular}{|c|c|c|c|c|}
\hline \multirow[b]{2}{*}{ Node } & \multirow[b]{2}{*}{ State } & \multicolumn{3}{|c|}{ Marginal Probabilities (in \%) } \\
\hline & & $\begin{array}{l}\text { Original } \\
\text { Network }\end{array}$ & $\begin{array}{l}\text { Learned } \\
\text { Network }\end{array}$ & Difference \\
\hline \multirow{2}{*}{ A } & TRUE & 81 & 80 & \multirow{2}{*}{1} \\
\hline & FALSE & 19 & 20 & \\
\hline \multirow{2}{*}{ B } & Class 1 & 22 & 24 & \multirow{2}{*}{2} \\
\hline & Class2 & 78 & 76 & \\
\hline \multirow{2}{*}{$\mathrm{C}$} & Yes & 43 & 46 & \multirow{2}{*}{3} \\
\hline & No & 57 & 54 & \\
\hline \multirow{2}{*}{$\mathrm{D}$} & State2 & 67 & 62 & \multirow{2}{*}{5} \\
\hline & State3 & 33 & 38 & \\
\hline \multirow{2}{*}{$\mathrm{E}$} & Right & 46 & 47 & \multirow{2}{*}{1} \\
\hline & Wrong & 54 & 53 & \\
\hline \multirow{2}{*}{$\mathrm{F}$} & Temp & 57 & 57 & \multirow{2}{*}{0} \\
\hline & Perm & 43 & 43 & \\
\hline $\mathrm{G}$ & State2 & 51 & 52 & 1 \\
\hline
\end{tabular}




\begin{tabular}{|c|c|c|c|c|}
\hline \multirow{2}{*}{ H } & State3 & 49 & 48 & \\
\hline & State2 & 52 & 53 & 1 \\
\cline { 2 - 5 } & State3 & 48 & 47 & 1 \\
\hline \multirow{2}{*}{ I } & State2 & 47 & 48 & \multirow{2}{*}{1} \\
\cline { 2 - 4 } & State3 & 53 & 52 & \\
\hline \multicolumn{4}{|c|}{ Average Difference $=1.666667$} \\
Variance Difference $=2.25$ \\
\hline
\end{tabular}

Table 4 represents the comparison between the sample network given in figure 1 and the network that is learned by CCGA BN-Constructor given in figure 3. Comparison is performed on the basis of marginal probabilities at each state of each node and as can be visible by the table the differences between the given and learned network are low on average the difference is 1.67 and on average the standard deviation is 1.5 for the difference between the probabilities. (Probabilities are given in percent)

Table 5: Comparison of the network 1 with the resultant network from the CCGA-BN Constructor on the basis of Marginal Probabilities when evidence is placed on I

\begin{tabular}{|c|c|c|c|c|}
\hline \multirow[b]{2}{*}{ Node } & \multirow[b]{2}{*}{ State } & \multicolumn{3}{|c|}{ Marginal Probabilities (in \%) } \\
\hline & & $\begin{array}{l}\text { Original } \\
\text { Network }\end{array}$ & $\begin{array}{l}\text { Learned } \\
\text { Network }\end{array}$ & Difference \\
\hline \multirow{2}{*}{ A } & TRUE & 81 & 79 & \multirow{2}{*}{2} \\
\hline & FALSE & 19 & 21 & \\
\hline \multirow{2}{*}{ B } & Class1 & 24 & 28 & \multirow{2}{*}{4} \\
\hline & Class2 & 76 & 72 & \\
\hline \multirow{2}{*}{$\mathrm{C}$} & Yes & 43 & 46 & \multirow{2}{*}{3} \\
\hline & No & 57 & 54 & \\
\hline \multirow{2}{*}{$\mathrm{D}$} & State2 & 67 & 62 & \multirow{2}{*}{5} \\
\hline & State3 & 33 & 38 & \\
\hline \multirow{2}{*}{$\mathrm{E}$} & Right & 46 & 47 & \multirow{2}{*}{1} \\
\hline & Wrong & 54 & 53 & \\
\hline \multirow{2}{*}{$\mathrm{F}$} & Temp & 57 & 57 & \multirow{2}{*}{0} \\
\hline & Perm & 43 & 43 & \\
\hline \multirow{2}{*}{ G } & State2 & 51 & 51 & \multirow{2}{*}{0} \\
\hline & State3 & 49 & 49 & \\
\hline $\mathrm{H}$ & State2 & 53 & 53 & 0 \\
\hline
\end{tabular}

\begin{tabular}{|c|c|c|c|c|}
\hline & State3 & 47 & 47 & \\
\hline I & \multicolumn{3}{|c|}{ Evidence on I at state 2 } \\
\hline \multicolumn{4}{|c|}{$\begin{array}{c}\text { Average Difference }=1.875 \\
\text { Variance Difference }=3.839286\end{array}$} \\
\end{tabular}

Table 6: Comparison of the network 1 with the resultant learned network from the CCGA-BN Constructor on the basis of Marginal Probabilities when evidence is placed on $I$ and $B$

\begin{tabular}{|c|c|c|c|c|}
\hline \multirow[b]{2}{*}{ Node } & \multirow[b]{2}{*}{ State } & \multicolumn{3}{|c|}{ Marginal Probabilities(in \%) } \\
\hline & & $\begin{array}{l}\text { Original } \\
\text { Network }\end{array}$ & $\begin{array}{c}\text { Learned } \\
\text { Network }\end{array}$ & Difference \\
\hline \multirow{2}{*}{ A } & TRUE & 73 & 69 & \multirow{2}{*}{4} \\
\hline & FALSE & 27 & 31 & \\
\hline B & \multicolumn{4}{|c|}{ Evidence on B at state class1 } \\
\hline \multirow{2}{*}{$\mathrm{C}$} & Yes & 44 & 40 & \multirow{2}{*}{4} \\
\hline & No & 56 & 60 & \\
\hline \multirow{2}{*}{$\mathrm{D}$} & State2 & 67 & 61 & \multirow{2}{*}{6} \\
\hline & State3 & 33 & 39 & \\
\hline \multirow{2}{*}{$\mathrm{E}$} & Right & 53 & 47 & \multirow{2}{*}{6} \\
\hline & Wrong & 47 & 53 & \\
\hline \multirow{2}{*}{$\mathrm{F}$} & Temp & 59 & 57 & \multirow{2}{*}{2} \\
\hline & Perm & 41 & 43 & \\
\hline \multirow{2}{*}{ G } & State2 & 51 & 52 & \multirow{2}{*}{1} \\
\hline & State3 & 49 & 48 & \\
\hline \multirow{2}{*}{$\mathrm{H}$} & State2 & 52 & 53 & \multirow{2}{*}{1} \\
\hline & State3 & 48 & 47 & \\
\hline I & \multicolumn{4}{|c|}{ Evidence on I at state 2} \\
\hline \multicolumn{5}{|c|}{ Average Difference $=3.428571$} \\
\hline \multicolumn{5}{|c|}{ Variance Difference $=4.619048$} \\
\hline
\end{tabular}

Table 7: Comparison of the network1 with the learned network from the CCGA-BN Constructor on the basis of Marginal Probabilities when evidence is placed on $I$ and B

\begin{tabular}{|c|c|c|c|c|}
\hline & & \multicolumn{3}{|c|}{ Marginal Probabilities(in \%) } \\
\hline Node & State & $\begin{array}{l}\text { Original } \\
\text { Network }\end{array}$ & $\begin{array}{l}\text { Learned } \\
\text { Network }\end{array}$ & Difference \\
\hline
\end{tabular}




\begin{tabular}{|c|c|c|c|c|}
\hline \multirow{2}{*}{ A } & TRUE & 72 & 69 & \multirow{2}{*}{3} \\
\hline & FALSE & 28 & 31 & \\
\hline B & \multicolumn{4}{|c|}{ Evidence on B at state class1 } \\
\hline $\mathrm{C}$ & \multicolumn{4}{|c|}{ Evidence on $\mathrm{C}$ at state Yes } \\
\hline \multirow{2}{*}{$\mathrm{D}$} & State2 & 55 & 61 & \multirow{2}{*}{6} \\
\hline & State3 & 45 & 39 & \\
\hline \multirow{2}{*}{$\mathrm{E}$} & Right & 52 & 48 & \multirow{2}{*}{4} \\
\hline & Wrong & 48 & 52 & \\
\hline \multirow{2}{*}{$\mathrm{F}$} & Temp & 59 & 56 & \multirow{2}{*}{3} \\
\hline & Perm & 41 & 44 & \\
\hline \multirow{2}{*}{$\mathrm{G}$} & Class2 & 55 & 55 & \multirow{2}{*}{0} \\
\hline & Class3 & 45 & 45 & \\
\hline \multirow{2}{*}{$\mathrm{H}$} & Class2 & 52 & 55 & \multirow{2}{*}{3} \\
\hline & Class3 & 48 & 45 & \\
\hline $\mathrm{I}$ & \multicolumn{4}{|c|}{ Evidence on I at state 2} \\
\hline \multicolumn{5}{|c|}{ Average Difference $=3.166667$} \\
\hline & Variar & Diffe & 3.76 & \\
\hline
\end{tabular}

The learned structure for the alarm network preserves 5 out of 47 parent-child relationships of the original network. The marginal probabilities,(in percent) calculated from the CPDs that are learned from data for the network produced by CCGA-BN Constructor, have low difference when compared with that of original network. The average standard deviation of the differences of the marginal probabilitiesof two networks is 3.56231 , which reflects that the resultant network learned performs almost similar to the original network when performing inference. When evidence is placed at state normal of node $\mathrm{ArtCO} 2$ the standard deviation of difference between marginal probabilities of learned and original alarm network is 2.773865 . When evidence is placed at state high of node PAP the standard deviation is 5.061452, and when evidence is at normal state of $\mathrm{SaO2the}$ differences are still found to be low which is reflected by the standard deviation which is found to be 4.349598 .

Time complexity is a challenge in solving the problem of learning network form data, the average time taken to by CCGA-BN Constructor for learning network from the data of network 1 is almost 15 minutes and for alarm network it was almost 5 hours when executed for dataset of 1000 records

\section{CONCLUSION}

We implemented CCGA-BN Constructor for achieving the task of Bayesian network learning from data sets. The approach implemented in this paper addressed the problem by decomposingit into two dependent subtasks that is to find an optimal nodes' ordering and an optimal connectivity matrix. We analyzed the performance of the algorithm on the basis of the differences in marginal probabilities between those that are learned from the data for the learned structure and those that are observed from the original network. The networks we analyzed in the paper are alarm network and another network of 9 nodes. The results showed that the solutions' obtained marginal probabilities (in percent) when compared to the original networks have low differences, on average difference is between 1 and 10. For the analysis data sets of 1000 instances are used for both the networks. Even after placing evidence on different nodes the inferred marginal probabilities have low variance/standard deviation and low average difference. But, time complexity of the solution is high and can be improved as it is a problem that is suitable for parallel computing environment as there are two independent species' populations that can be constructed side by side.

\section{REFERENCES}

[1] D. Heckerman, D. Geiger, and D. M. Chickering. "Learning Bayesian networks: The combination ofknowledge and statistical data". Machine Learning, 20:197-243, 1995.

[2] G. F. Cooper and E. Herskovits. "A Bayesian method for the induction of probabilistic networks from data".Machine Learning, 9:309-347, 1992.

[3] D. M. Chickering, D. Geiger, and D. Heckerman. "Learning Bayesian Networks is NP-Hard". Technical report, Microsoft Research, 1994.

[4] C. Cotta and J. Muruzabal. "On the learning of Bayesian network graph structures via evolutionary programming". In Proceedings of the 2nd European Workshop on Probabilistic Graphical Models, pages 6572,2004

[5] P. Larranaga and M. Poza. "Structure learning of bayesian networks by genetic algorithms: A performance analysis of control parameters". IEEE Journal on Pattern Analysis and Machine Intelligence, 18(9):912-926, 1996

[6] M. Wong, W. Lam, and K. Leung. "Using evolutionary programming and minimum description length principle for data mining of Bayesian networks". IEEE Transactions PAMI, 21(2):174-178, 1999.

[7] M. L. Wong, S. Y. Lee, and K.-S.Leung. "A hybrid data mining approach to discover bayesian networks using evolutionary programming”. In GECCO, pages 214$222,2002$.

[8] Arthur Carvalho, David Cheriton. "A Cooperative Coevolutionary Genetic Algorithm for Learning Bayesian Network Structures". School of Computer Science, University of Waterloo, Ontario, Canada. July 2011.

[9] D. Koller and N. Friedman. "Probabilistic Graphical Models: Principles and Techniques”. MIT Press, 2009.

[10] Spirtes, Glymour, AndScheines. "Causation, Prediction And Search”. Springer-Verlag, 1993.

[11] R. W. Robinson. "Counting Unlabeled Acyclic Digraphs". In Combinatorial Mathematics V, volume 622 of Lecture Notes in Mathematics, pages 28 43,1977 .

[12] M. Potter and K. De Jong."A cooperative coevolutionary approach to function optimization". In Third Conference on Parallel Problem Solving from Nature, pages 249-257, 1994.

[13] A. Delaplace, T. Brouard, and H. Cardot."Two Evolutionary Methods for Learning Bayesian Network Structure"s. In Computational Intelligence and 
International Journal of Computer Applications (0975 - 8887)

Volume 115 - No. 20, April 2015

Security, pages 288-297. 2007.

[14] Mitchell A. Potter and Kenneth A. De Jong " $A$ Cooperative Coevolutionary Approach to Function Optimization".Computer Science Department, George Mason University, Fairfax, VA 22030, USA.
[15] J. Pearl. "Probabilistic reasoning in intelligent systems: networks of plausible inference". Morgan Kaufmann, 1997.

[16] Alexandra M. Carvalho "Scoring functions for learning Bayesian networks”.Inesc-id Tec. Rep, 2009 